\title{
Aplicativo móvel: intervenções fisioterapêuticas à idosos frágeis
}

\author{
Mobile application: physical therapy interventions in frail elderly \\ Aplicación móvil: intervenciones fisioterapéuticas a personas mayores frágiles \\ Wagner Elias de Melo Moreira', Gustavo Detomi Rodrigues², Jorge Luiz de Carvalho Mello ${ }^{3}$, \\ Paulo Roberto Maia ${ }^{4}$, Diba Maria Sebba Tosta de Souza ${ }^{5}$
}

RESUMO I O objetivo deste estudo foi construir e validar um algoritmo e desenvolver um software do tipo aplicativo móvel para o diagnóstico multidimensional da vulnerabilidade clínico funcional e tratamento fisioterapêutico em idosos. Trata-se de um estudo analítico, observacional, aplicado na modalidade de produção tecnológica, baseado na engenharia de software e fundamentada no design centrado no usuário. O algoritmo foi elaborado a partir de estudos em bases de dados da área da saúde, validado por 13 fisioterapeutas e a construção do software deu-se através da linguagem JAVA, a partir da conversão do algoritmo. Para validação do conteúdo do algoritmo, foi utilizada a técnica Delphi e análise estatística do Índice de Validade de Conteúdo (IVC). O consenso foi atingido logo na primeira rodada de avaliação, a concordância entre juízes pelo IVC foi de 1,0. 0 aplicativo móvel Gerontofisio foi construido para uso em smartphones e tablets, registrado no Instituto Nacional da Propriedade Industrial número: BR512020002269-3 e possui 34 telas que orientam fisioterapeutas gerontólogos nas tomadas de decisão, servindo como ferramenta de apoio nos campos de aplicação em saúde, administração sanitária, doença, assistência médica e terapia diagnóstica. Dessa forma, o algoritmo foi construído e validado, o software do tipo aplicativo móvel para o diagnóstico multidimensional da vulnerabilidade clínico funcional e tratamento fisioterapêutico em idosos foi desenvolvido, é válido na área de fisioterapia e deve contribuir para fisioterapeutas e pessoas idosas.
Descritores | Aplicativos Móveis; Geriatria; Idoso Fragilizado; Fisioterapeutas.

ABSTRACT I The aim of this study was to build and validate an algorithm and develop a software for the multidimensional diagnosis of functional clinical vulnerability and physical therapy for older adults. This is an analytical and observational study applied in technological production, based on software engineering and user-centered design. The algorithm was developed based on studies in health databases, validated by 13 physical therapists and the software was built using the JAVA language from the algorithm's conversion. The Delphi technique and statistical analysis via the Content Validity Index (CVI) were used to validate the algorithm. The consensus was reached in the first evaluation round and the agreement between judges by the CVI was 1.0. The "Gerontofisio" mobile application was built for use on smartphones and tablets and registered at the Brazilian National Institute of Industrial Property number: BR512020002269-3; the application consists of 34 screens that guide gerontological physical therapists in decision making, serving as a support tool in the applied fields in health, health administration, illness, medical assistance and diagnostic therapy. The algorithm was built and validated, the mobile application software for the multidimensional diagnosis of clinical functional vulnerability and physical therapy treatment in older adults was developed and is valid in the field of physical therapy and should contribute to physical therapists and older adults.

'Universidade do Vale do Sapucaí (UNIVAS) - Pouso Alegre (MG), Brasil. E-mail: wagner.fisioterapia@yahoo.com.br. ORCID-0000-0002-8287-7476

2Universidade Federal de São João del-Rei (UFSJ) - São João del-Rei (MG), Brasil. E-mail: detomigustavo@gmail.com. ORCID-0000-0001-8382-3368

3Universidade do Vale do Sapucaí (UNIVAS) - Pouso Alegre (MG), Brasil. E-mail: jorgeluis_melo@yahoo.com.br. ORCID-0000-0002-2206-4211

${ }^{4}$ Universidade do Vale do Sapucaí (UNIVAS) - Pouso Alegre (MG), Brasil. E-mail: prmmaia2012@gmail.com. ORCID-0000-0003-0044-195X

5 Universidade do Vale do Sapucaí (UNIVAS) - Pouso Alegre (MG), Brasil. E-mail: souzadiba@gmail.com. ORCID-0000-0002-4743-2455 
Keywords I Mobile Applications; Geriatrics; Frail Older Adults; Physical Therapists.

RESUMEN | El objetivo de este estudio fue construir y validar un algoritmo y desarrollar un software de aplicación móvil para el diagnóstico multidimensional de la vulnerabilidad funcional clínica y el tratamiento de fisioterapia en ancianos. Es un estudio analítico, observacional, aplicado en la modalidad de producción tecnológica, basado en la ingeniería de software y basado en el Diseño Centrado en el Usuario. El algoritmo se desarrolló a partir de estudios en bases de datos del área de salud, validados por 13 fisioterapeutas y la construcción del software se realizó a través del lenguaje JAVA, a partir de la conversión del algoritmo. Para validar el contenido del algoritmo se utilizó la técnica Delphi y el análisis estadístico del Índice de Validez de Contenido (IVC). Se alcanzó consenso en la primera ronda de evaluación, el acuerdo entre jueces para el IVC fue 1.0. La aplicación móvil Gerontofisio fue construida para su uso en teléfonos inteligentes y tabletas, registrada en el Instituto Nacional de la Propiedad Industrial número: BR512020002269-3, cuenta con 34 pantallas que guían a los fisioterapeutas gerontólogos en la toma de decisiones, sirviendo como herramienta de apoyo en los campos de aplicación en Salud, Administración de la salud, Enfermedad, Atención médica y Terapia diagnóstica. Así, se construyó y validó el algoritmo, se desarrolló el software de aplicación móvil para el diagnóstico multidimensional de la vulnerabilidad funcional clínica y el tratamiento de fisioterapia en ancianos, que es válido en el campo de la fisioterapia y debe contribuir a fisioterapeutas y personas mayores.

Palabras clave | Aplicaciones Móviles; Geriatría; Anciano Frágil; Fisioterapeutas.

\section{INTRODUÇÃO}

O processo de transição demográfica determinou mudanças no padrão de adoecimento da população ${ }^{1}$. Por isso, as escalas de avaliação das atividades diárias são usualmente utilizadas, por serem instrumentos rápidos de avaliação, triagem e estratificação de risco ${ }^{2}$, apontando o grau de independência de um indivíduo na realização destas atividades e, indiretamente, o estado de saúde e a necessidade de auxílio ${ }^{3}$.

A avaliação geriátrica ampla (AGA) é uma das principais ferramentas utilizadas para identificar o idoso frágil, por permitir um processo diagnóstico global e amplo, envolvendo o paciente e sua família, com a finalidade de verificar a saúde do idoso como um todo ${ }^{4}$. Entretanto, pode-se considerá-la como um procedimento diagnóstico demorado, que apresenta uma relação custo-benefício indesejada em saúde pública 5 .

Pensando nisso, cientistas brasileiros desenvolveram o índice de vulnerabilidade clínico funcional-20 (IVCF20) como ferramenta de rastreio de idosos frágeis de fácil aplicação na atenção primária ${ }^{5}$. Além da AGA e do IVCF20, outros instrumentos são utilizados por fisioterapeutas na avaliação funcional de idosos, como a escala de Tinneti e o timed up and go, que fornecem informações sobre a mobilidade e o risco de quedas ${ }^{6,7}$; o índice de Barthel e a escala de Katz, que mensuram a execução das atividades básicas da vida diária ${ }^{3,8}$; a escala de Lawton para as atividades instrumentais da vida diária ${ }^{9}$; e o physical performance test modificado, para avaliar o nível de funcionalidade física ${ }^{10}$.
No entanto, existe necessidade de propor soluções que preservem a biodiversidade, incentivando a substituição do uso de papéis por softwares ${ }^{11}$. Além disso, as ferramentas digitais geram maior segurança na informação, bem como o papel atualmente é considerado um veículo de transmissão do Sars-CoV-2 (covid-19), devido ao coronavírus persistir entre quatro e cinco dias nessa superfície ${ }^{12}$.

Desta maneira, há uma preocupação maior com os idosos, por terem maiores chances de irem a óbitos em decorrência das comorbidades associadas ao processo de fragilização ${ }^{13}$. Sendo assim, a população idosa representa a necessidade de novas perspectivas na saúde, em que a gerontecnologia pode ser útil para avaliar, diagnosticar e auxiliar na tomada de decisão do profissional ${ }^{14}$.

A gerontecnologia é o estudo multidisciplinar do envelhecimento e da tecnologia para reduzir os anos de morbidade e desconforto, aumentando, consequentemente, a autonomia funcional de idosos ${ }^{14}$. Desse modo, diversos aplicativos móveis são descritos na literatura para beneficiar a saúde e o cuidado de idosos, com resultados positivos quanto às suas funcionalidades ${ }^{15}$. Entretanto, muitos desses aplicativos são utilizados por idosos e seus cuidadores, evidenciando a necessidade de novos apps sob gerenciamento e supervisão de profissionais da área.

Os aplicativos móveis são conceituados como um conjunto de ferramentas desenhado para realizar tarefas e trabalhos específicos, com bom desempenho, facilidade de transporte e armazenamento ${ }^{16}$. $\mathrm{Na}$ área de saúde, podem apresentar funcionalidades capazes de melhorar a 
acessibilidade a tratamentos, fornecendo de forma rápida e com exatidão os diagnósticos ${ }^{15,17}$.

No entanto, na área de fisioterapia gerontológica, esses aplicativos requerem uma especificidade, com aplicações técnicas voltadas para a triagem do idoso em situação de vulnerabilidade clínico funcional, prevenção e reabilitação de déficits funcionais ocasionados pelo envelhecimento ou por outros fatores decorrentes da senilidade ${ }^{18,19}$.

Considerando-se a definição do estrato de vulnerabilidade clínico funcional sugerida por Moraes et al. ${ }^{5}$, idosos apresentam, em decorrência do nível de funcionalidade, fatores de risco, doenças e comorbidades múltiplas, além de uma heterogeneidade funcional, com complexidade clínica e necessidade de acompanhamento gerontogeriátrico especializado diferentes.

Portanto, por haver a necessidade de avaliar constantemente as condições de saúde dos idosos pelo fisioterapeuta, profissional de saúde que busca manter a funcionalidade de órgãos e sistemas, e de incentivar a preservação aos recursos naturais, este estudo tem como objetivo construir e validar um algoritmo e desenvolver um software do tipo aplicativo móvel para o diagnóstico multidimensional da vulnerabilidade clínico funcional e tratamento fisioterapêutico em idosos.

\section{METHODOLOGY}

Trata-se de um estudo observacional, analítico e transversal aplicado na modalidade de produção tecnológica, baseado na engenharia de software e fundamentado no Design Centrado no Usuário. $\mathrm{O}$ pesquisador e os participantes obedeceram aos termos contidos na Resolução do Conselho Nacional de Saúde (CNS) 466/2012, e os materiais e informações obtidos foram utilizados, único e exclusivamente, para os objetivos dessa pesquisa.

Para compor a amostra dos juízes do algoritmo, optouse por seguir as recomendações de Haynes, Richard e Kubany ${ }^{20}$, que sugerem entre seis e vinte juízes. Sendo assim, a população de juízes deste estudo constou de treze profissionais fisioterapeutas, selecionados por conveniência e que assinaram o termo de consentimento livre e esclarecido (TCLE). Foram inclusos profissionais fisioterapeutas graduados há no mínimo cinco anos, com titulação mínima de especialização. Foram excluídos fisioterapeutas com registro inativo no Conselho Regional de Fisioterapia e Terapia Ocupacional ou que recusaram participar da pesquisa após assinatura do TCLE.

\section{Construção e validação do algoritmo}

A elaboração do algoritmo se dividiu em duas etapas: a primeira correspondeu ao levantamento do conteúdo por revisão integrativa da literatura relacionada a funcionalidade de idosos, que reuniu 43 artigos e uma diretriz. Foram selecionados estudos nos idiomas inglês, português e espanhol, utilizando-se os descritores: Idoso Fragilizado; Equilíbrio Postural; Análise da Marcha; Avaliação Geriátrica; Atividades Cotidianas; Terapia por Exercício, disponíveis no site dos Descritores em Ciências da Saúde. Foram excluídos os capítulos de livros, teses, dissertações, monografias e artigos que, após leitura do resumo, não atendiam os objetivos propostos.

As bases de dados eletrônicas consultadas foram a Literatura Latino-Americana e do Caribe em Ciências da Saúde (LILACS), Medical Literature Analysis and Retrievel System Online (MEDLINE) e Scientific Eletronic Livrary Online (SciELO), sem delimitação temporal, pois a intenção era selecionar estudos que atendessem aos objetivos pré-estabelecidos.

Os estudos foram classificados quanto ao nível de evidência, seguindo as recomendações de Melnyk e Fineout-Overholt ${ }^{21}$, sendo nível I aquelas oriundas de revisão sistemática ou meta-análise de ensaios clínicos randomizados controlados relevantes ou de diretrizes clínicas baseadas em estudo clínico randomizado; nível II, as derivadas de pelo menos um ensaio clínico randomizado; nível III, as obtidas de ensaios clínicos bem delineados sem randomização; nível IV, provenientes de estudos de coorte e de caso-controle bem delineados; nível $\mathrm{V}$, as originárias de revisão sistemática de estudos descritivos e qualitativos; nível VI, provenientes de um único estudo descritivo ou qualitativo; e por fim, nível VII, as evidências oriundas de opinião de autoridades e/ ou relatório de comitês de especialistas.

A classificação dos estudos segundo estas recomendações possibilita auxiliar o profissional na avaliação crítica de pesquisas e, consequentemente, na tomada de decisão sobre a incorporação das evidências à prática clínica.

Já a segunda etapa correspondeu à formulação e à montagem do algoritmo, que contou com auxílio da plataforma virtual Lucidchart ${ }^{\circledR}$. O algoritmo possui dois sistemas de avaliação, em que um é amplo e o outro, específico da área de fisioterapia. Na avaliação ampla, foram descritos aspectos multidimensionais da condição de saúde do idoso. $\mathrm{Na}$ avaliação fisioterapêutica, foram descritas condições relacionadas às disfunções que envolvem os sistemas musculoesquelético e respiratório. 
O sequenciamento das informações foi planejado no sentido de identificar primeiro aqueles idosos que estão em risco para declínio funcional, vulnerabilidade e/ou fragilidade (avaliação ampla), bastando, para isso, duas ou mais respostas positivas nesta avaliação. Após essa etapa, é possível direcionar o idoso a serviços de acompanhamento da atenção básica ou realizar uma avaliação fisioterapêutica gerontológica (avaliação específica).

No caso de uma resposta positiva na avaliação fisioterapêutica, o algoritmo direciona para sugestão de objetivos de reabilitação. Caso não seja apresentada nenhuma resposta positiva, o profissional é orientado a reavaliar e/ou encaminhar o idoso às outras especialidades da área de saúde.

A validação do instrumento por juízes aconteceu por meio da aplicação de um questionário criado pelos autores, que possuem formação na área de saúde e atuam em geriatria-gerontologia. $\mathrm{O}$ questionário é composto pelos itens do algoritmo e o seu julgamento foi com uso de uma escala tipo Likert, permitindo verificar a concordância dos fisioterapeutas sobre as informações que auxiliam na avaliação e na identificação do risco para declínio funcional, vulnerabilidade e/ou fragilidade em idosos e orientação da classe profissional sobre condutas aplicáveis a esta população. Para o processo de validação, foram convidados por meio de carta convite enviada via e-mail, 17 fisioterapeutas que atuam na área de gerontologia em Instituições de Ensino Superior e em Instituições de Longa Permanência para Idosos (ILPI). Aos que aceitaram, foi realizado a abordagem presencial pelo pesquisador para apresentação do TCLE e, posteriormente, a disponibilização do algoritmo e do seu questionário.

Após a validação do instrumento, foi realizado um estudo piloto que testou a adequabilidade do algoritmo em dez idosos da comunidade.

\section{Construção do aplicativo móvel}

Para construir um aplicativo móvel é necessário um algoritmo para orientar o profissional da área de tecnologia da informação. Após o algoritmo do estudo ter sido validado, ele foi convertido para uma linguagem de programação traduzida em linhas de códigos por um profissional graduado na área de ciência da computação. Essa tradução permite o desenvolvimento do software do tipo aplicativo móvel para uso em aparelhos celulares e tablets. Optou-se pelo design centrado no usuário, em que o pesquisador foi consultado pelo profissional da área de computação quanto às necessidades dos fisioterapeutas para aplicar as avaliações do algoritmo e convidado a participar dos testes de usabilidade do sistema desenvolvido.
Após a conversão do algoritmo em aplicativo móvel, foram realizados três testes com o software. No primeiro teste, foram testadas as avaliações do algoritmo e solicitada a criação de um menu em que o profissional fisioterapeuta pudesse escolher em aplicar de forma rápida a avaliação no idoso (avaliação expressa) ou cadastrá-lo e manter suas avaliações e informações pessoais salvas (avaliação cadastrada). Além disso, foram adicionadas as seções de telefones de emergência para uso em caso de intercorrências, o algoritmo em formato de imagem, as referências utilizadas e o nome dos proprietários do aplicativo móvel.

No segundo teste, foram testadas as novas funcionalidades, e solicitada a correção de alguns erros ortográficos e a inclusão da logomarca do aplicativo na tela inicial. Por fim, no terceiro teste, foram testadas todas as ferramentas, sem novas solicitações.

O aplicativo móvel é de uso off-line e dispensa a criação de perfis ou contas de acesso específico. Foi desenvolvido em Android Package (arquivo de pacote destinado ao sistema operacional Android da Google ${ }^{\circledR}$ ) $e$ a engenharia de software realizada foi através da linguagem de programação Java, com interface gráfica em XML, usando o IDE Android Studio, arquitetura Model View Controller, banco de dados SQLite e a técnica Scrum.

\section{Análise estatística}

Para a validação do questionário como um todo, foi calculado o índice de validade de conteúdo (IVC), cujo resultado necessita ser superior a 0,90 (> 90\%) para ser considerado validado. Sua finalidade foi medir a porcentagem de juízes que estiveram em concordância sobre determinados aspectos do instrumento e de seus itens ${ }^{22}$. Foi empregado uma escala tipo Likert com pontuações que variam entre um e quatro: 4=totalmente adequado; 3=Adequado; 2 =parcialmente adequado; e 1=inadequado. O escore do IVC foi calculado por meio da soma de concordância dos itens que foram marcados por " 4 " ou “3” pelos 13 juízes.

A técnica Delphi também foi empregada, para buscar o consenso entre os juízes nas respostas "Totalmente adequada" e "Adequada"23. Utilizou-se os programas Minitab versão 18.1 e Statistical Package for the Social Sciences, versão 22.0.

\section{RESULTADOS}

Por meio da literatura pesquisada, construiu-se o algoritmo conforme a Figura 1. 


\section{ALGORITMO PARA DIAGNÓSTICO MULTIDIMENSIONAL DA VULNERABILIDADE CLINICO FUNCIONAL E TRATAMENTO FISIOTERAPÊUTICO EM IDOSOS}

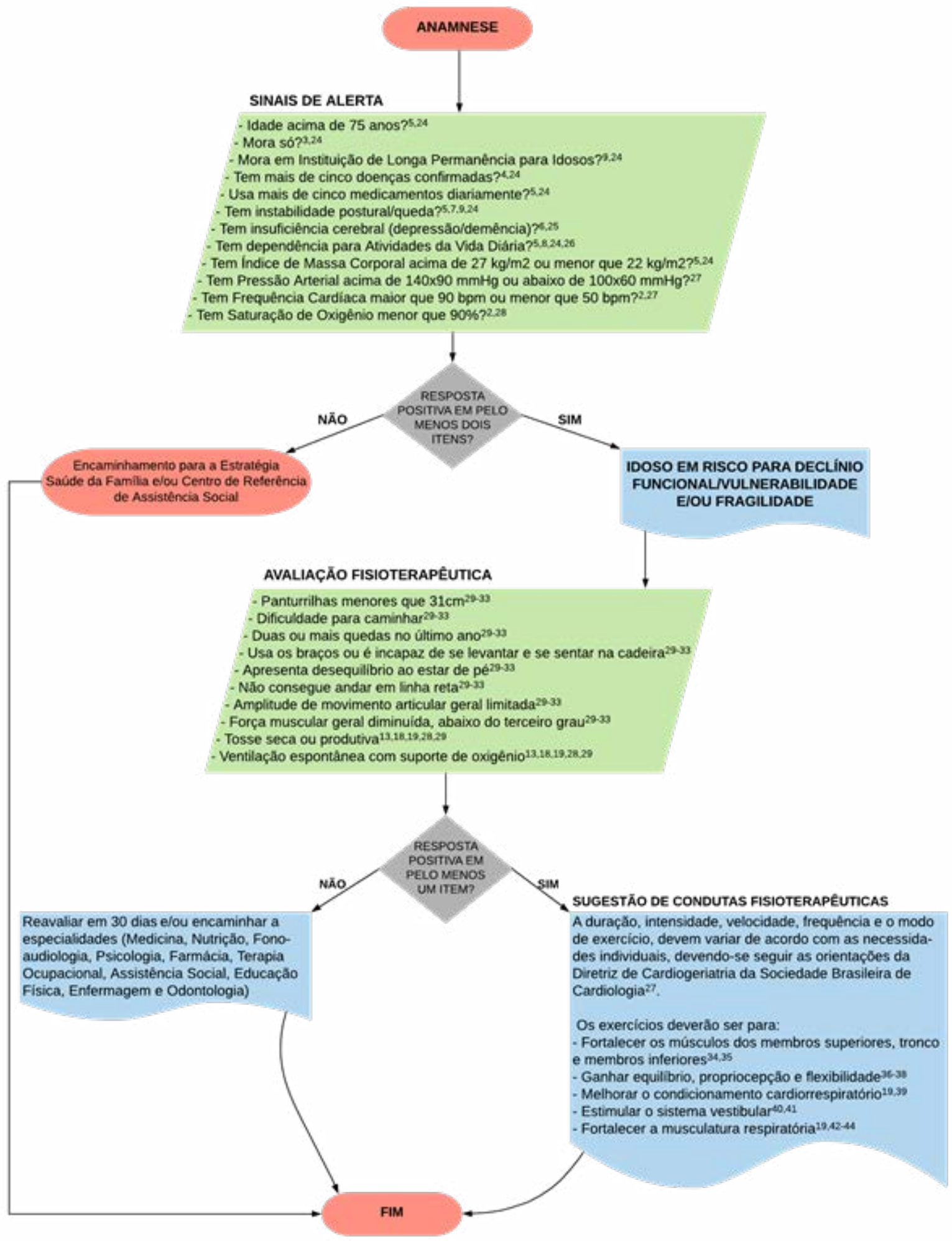

Figura 1. Algoritmo para diagnóstico multidimensional da vulnerabilidade clínico funcional e tratamento fisioterapêutico em idosos 
A avaliação dos estudos quanto ao nível de evidência seguiu as recomendações de Melnyk e Fineout-Overholt ${ }^{21}$, como demonstrado na Tabela 1.

Tabela 1. Nível de evidência dos estudos do algoritmo, Pouso Alegre (MG), Brasil, 2020

\begin{tabular}{|c|c|}
\hline Estudos & Nível de evidência \\
\hline Minosso et al. ${ }^{3}$ & 4 \\
\hline Saraiva et al. ${ }^{4}$ & 6 \\
\hline Moraes et al. ${ }^{5}$ & 4 \\
\hline Piovesan et al. ${ }^{6}$ & 6 \\
\hline Alexandre et al. ${ }^{7}$ & 6 \\
\hline Rebustini et al. ${ }^{8}$ & 6 \\
\hline Wu et al. ${ }^{13}$ & 6 \\
\hline Pillatt, Nielsson e Schneider ${ }^{18}$ & 1 \\
\hline Moreira et al. ${ }^{19}$ & 4 \\
\hline Faller et al. ${ }^{24}$ & 1 \\
\hline Folstein, Folstein e McHugh ${ }^{25}$ & 4 \\
\hline Katz et al. ${ }^{26}$ & 4 \\
\hline Feitosa-Filho et al. ${ }^{27}$ & 7 \\
\hline Mendes et al. ${ }^{28}$ & 4 \\
\hline Cordeiro et al..$^{29}$ & 4 \\
\hline Giaquini, Lini e Doring ${ }^{30}$ & 4 \\
\hline Bushatsky et al. ${ }^{31}$ & 6 \\
\hline Clares, Freitas e Borges ${ }^{32}$ & 4 \\
\hline Alexandre et al. ${ }^{33}$ & 4 \\
\hline Soukkio et al. ${ }^{34}$ & 2 \\
\hline Carral et al..$^{35}$ & 2 \\
\hline Yamamoto et al. ${ }^{36}$ & 2 \\
\hline Rodriguez-Larrad et al. ${ }^{37}$ & 2 \\
\hline Criekinge et al.38 & 2 \\
\hline Allison, Brooke-Wavell e Folland ${ }^{39}$ & 2 \\
\hline Jia et al..$^{40}$ & 2 \\
\hline Vugt et al. ${ }^{41}$ & 2 \\
\hline Marioni et al. ${ }^{42}$ & 2 \\
\hline Fonseca et al. ${ }^{43}$ & 3 \\
\hline Marco et al. ${ }^{44}$ & 5 \\
\hline
\end{tabular}

Todos os juízes concordaram com a aplicabilidade do algoritmo, sendo demonstrado a concordância pelo Índice de Validade de Conteúdo na Tabela 2.

Por meio da análise, observa-se que o consenso foi atingido logo na primeira rodada de avaliação; nenhuma questão recebeu pontuação igual ou menor que dois (parcialmente adequada ou inadequada) e não foram apresentadas sugestões e opiniões pelos juízes. Sendo assim, o questionário não necessitou de uma segunda rodada para avaliação. Os dados são apresentados na Tabela 3.
Tabela 2. Itens do questionário julgados pelos juízes e Índice de Validade de Conteúdo, Pouso Alegre (MG), Brasil, 2020

Item de julgamento

Dimensão "Sinais de alerta"

- Idade acima de 75 anos

- Mora só?

- Mora em Instituição de Longa Permanência para Idosos? $\quad$ 1,0

- Tem mais de cinco doenças confirmadas?

- Usa mais de cinco medicamentos diariamente?

- Tem instabilidade postural/queda?

- Tem insuficiência cerebral (depressão/demência)?

- Tem dependência para Atividades da Vida Diária?

- Tem Índice de Massa Corporal acima de 27 kg/m2 ou menor que $22 \mathrm{~kg} / \mathrm{m} 2$ ?

- Tem Pressão Arterial acima de $140 \times 90$ mmHg ou abaixo de $100 \times 60 \mathrm{mmHg}$ ?

- Tem Frequência Cardíaca maior que 90 bpm ou menor que 50 bpm?

- Tem Saturação de Oxigênio menor que 90\%?

Dimensão "Resposta da avaliação geral"

- Encaminhamento para a Estratégia Saúde da Família e/ou

Centro de Referência de Assistência Social

- Idoso em risco para declínio funcional/vulnerabilidade e/ou fragilidade

Dimensão "Avaliação fisioterapêutica"

- Panturrilhas menores que $31 \mathrm{~cm}$

- Dificuldade para caminhar

- Duas ou mais quedas no último ano

- Usa os braços ou é incapaz de se levantar e se sentar na cadeira

- Apresenta desequilíbrio ao estar de pé

- Não consegue andar em linha reta

- Amplitude de movimento articular geral limitada

- Força muscular geral diminuída, abaixo do terceiro grau

- Tosse seca ou produtiva

- Ventilação espontânea com suporte de oxigênio

Dimensão "Resposta da avaliação específica"

- Reavaliar em 30 dias e/ou encaminhar a especialidades (Medicina, Nutrição, Fonoaudiologia, Psicologia, Farmácia,

Terapia Ocupacional, Assistência Social, Educação Física,

Enfermagem e Odontologia)

Sugestão de condutas fisioterapêuticas:

- Fortalecer os músculos dos membros superiores, tronco e membros inferiores

- Ganhar equilíbrio, propriocepção e flexibilidade

- Melhorar o condicionamento cardiorrespiratório

- Estimular o sistema vestibular

- Fortalecer a musculatura respiratória

Opinião "Apresentação geral do Algoritmo"

- Quanto à facilidade de leitura, clareza e compreensão das informações

- Quanto a sequência das informações

- Quanto ao layout/cor

Opinião do profissional

- O algoritmo é adequado para os fisioterapeutas?

Escore total

IVC Geral $>0,90=$ Validado 
Tabela 3. Avaliação do conteúdo dos itens do algoritmo pela técnica Delphi, Pouso Alegre, Minas Gerais, Brasil, 2020

\begin{tabular}{|c|c|c|c|c|c|c|}
\hline \multicolumn{7}{|l|}{$\begin{array}{l}\text { Técnica Delphi } \\
\text { Avaliação }\end{array}$} \\
\hline \multirow[t]{2}{*}{ Questões } & \multicolumn{2}{|c|}{ Adequada } & \multicolumn{2}{|c|}{$\begin{array}{l}\text { Totalmente } \\
\text { Adequada }\end{array}$} & \multicolumn{2}{|c|}{ Total } \\
\hline & $\mathrm{N}$ & $\%$ & $\mathbf{N}$ & $\%$ & $\mathrm{~N}$ & $\%$ \\
\hline $\begin{array}{l}\text { Dimensão "Sinais de alerta" } \\
\text { - Idade acima de } 75 \text { anos } \\
\text { - Mora só? } \\
\text { - Mora em Instituição de Longa Permanência para Idosos? } \\
\text { - Tem mais de cinco doenças confirmadas? } \\
\text { - Usa mais de cinco medicamentos diariamente? } \\
\text { - Tem instabilidade postural/queda? } \\
\text { - Tem insuficiência cerebral (depressão/demência)? } \\
\text { - Tem dependência para Atividades da Vida Diária? } \\
\text { - Tem Índice de Massa Corporal acima de } 27 \mathrm{~kg} / \mathrm{m} 2 \text { ou menor que } 22 \mathrm{~kg} / \mathrm{m} 2 \text { ? } \\
\text { - Tem Pressão Arterial acima de } 140 \times 90 \mathrm{mmHg} \text { ou abaixo de } 100 \times 60 \mathrm{mmHg} \text { ? } \\
\text { - Tem Frequência Cardíaca maior que } 90 \text { bpm ou menor que } 50 \text { bpm? } \\
\text { - Tem Saturação de Oxigênio menor que } 90 \% \text { ? }\end{array}$ & $\begin{array}{l}05 \\
05 \\
05 \\
05 \\
05 \\
05 \\
05 \\
05 \\
05 \\
05 \\
05 \\
05\end{array}$ & $\begin{array}{l}38,5 \\
38,5 \\
38,5 \\
38,5 \\
38,5 \\
38,5 \\
38,5 \\
38,5 \\
38,5 \\
38,5 \\
38,5 \\
38,5\end{array}$ & $\begin{array}{l}08 \\
08 \\
08 \\
08 \\
08 \\
08 \\
08 \\
08 \\
08 \\
08 \\
08 \\
08\end{array}$ & $\begin{array}{l}61,5 \\
61,5 \\
61,5 \\
61,5 \\
61,5 \\
61,5 \\
61,5 \\
61,5 \\
61,5 \\
61,5 \\
61,5 \\
61,5\end{array}$ & $\begin{array}{l}13 \\
13 \\
13 \\
13 \\
13 \\
13 \\
13 \\
13 \\
13 \\
13 \\
13 \\
13\end{array}$ & $\begin{array}{l}100 \\
100 \\
100 \\
100 \\
100 \\
100 \\
100 \\
100 \\
100 \\
100 \\
100 \\
100\end{array}$ \\
\hline $\begin{array}{l}\text { Dimensão "Resposta da avaliação geral" } \\
\text { • Encaminhamento para a Estratégia Saúde da Família e/ou Centro de Referência } \\
\text { de Assistência Social }\end{array}$ & 07 & 53,8 & 06 & 46,2 & 13 & 100 \\
\hline • Idoso em risco para declínio funcional/vulnerabilidade e/ou fragilidade & 05 & 38,5 & 08 & 61,5 & 13 & 100 \\
\hline $\begin{array}{l}\text { Dimensão "Avaliação fisioterapêutica" } \\
\text { - Panturrilhas menores que } 31 \text { cm } \\
\text { - Dificuldade para caminhar } \\
\text { - Duas ou mais quedas no último ano } \\
\text { - Usa os braços ou é incapaz de se levantar e se sentar na cadeira } \\
\text { - Apresenta desequilíbrio ao estar de pé } \\
\text { - Não consegue andar em linha reta } \\
\text { - Amplitude de movimento articular geral limitada } \\
\text { - Força muscular geral diminuída, abaixo do terceiro grau } \\
\text { - Tosse seca ou produtiva } \\
\text { - Ventilação espontânea com suporte de oxigênio } \\
\text { Dimensão "Resposta da avaliação específica" } \\
\text { - Reavaliar em } 30 \text { dias e/ou encaminhar a especialidades (Medicina, Nutrição, } \\
\text { Fonoaudiologia, Psicologia, Farmácia, Terapia Ocupacional, Assistência Social, } \\
\text { Educação Física, Enfermagem e Odontologia) }\end{array}$ & $\begin{array}{l}06 \\
06 \\
06 \\
06 \\
04 \\
05 \\
06 \\
00 \\
08 \\
09\end{array}$ & $\begin{array}{r}46,2 \\
46,2 \\
46,2 \\
46,2 \\
30,7 \\
38,5 \\
46,2 \\
00 \\
61,5 \\
69,3\end{array}$ & $\begin{array}{l}07 \\
07 \\
07 \\
07 \\
09 \\
06 \\
05 \\
13 \\
05 \\
04\end{array}$ & $\begin{array}{r}53,8 \\
53,8 \\
53,8 \\
53,8 \\
69,3 \\
61,5 \\
53,8 \\
100 \\
38,5 \\
30,7\end{array}$ & $\begin{array}{l}13 \\
13 \\
13 \\
13 \\
13 \\
13 \\
13 \\
13 \\
13 \\
13\end{array}$ & $\begin{array}{l}100 \\
100 \\
100 \\
100 \\
100 \\
100 \\
100 \\
100 \\
100 \\
100\end{array}$ \\
\hline $\begin{array}{l}\text { Sugestão de condutas fisioterapêuticas: } \\
\text { - Fortalecer os músculos dos membros superiores, tronco e membros inferiores } \\
\text { - Ganhar equilíbrio, propriocepção e flexibilidade } \\
\text { - Melhorar o condicionamento cardiorrespiratório } \\
\text { - Estimular o sistema vestibular } \\
\text { - Fortalecer a musculatura respiratória }\end{array}$ & $\begin{array}{c}11 \\
12 \\
04 \\
03 \\
11\end{array}$ & $\begin{array}{l}84,6 \\
92,3 \\
30,7 \\
23,0 \\
84,6\end{array}$ & $\begin{array}{r}01 \\
09 \\
10 \\
02\end{array}$ & $\begin{array}{r}7,7 \\
69,3 \\
77,0 \\
15,4\end{array}$ & $\begin{array}{l}13 \\
13 \\
13 \\
13\end{array}$ & $\begin{array}{l}100 \\
100 \\
100 \\
100\end{array}$ \\
\hline $\begin{array}{l}\text { Opinião "Apresentação geral do Algoritmo" } \\
\text { - Quanto à facilidade de leitura, clareza e compreensão das informações } \\
\text { - Quanto a sequência das informações } \\
\text { - Quanto ao layout/cor }\end{array}$ & $\begin{array}{r}01 \\
08\end{array}$ & $\begin{array}{r}7,7 \\
61,5\end{array}$ & $\begin{array}{r}12 \\
05\end{array}$ & $\begin{array}{l}92,3 \\
38,5\end{array}$ & $\begin{array}{l}13 \\
13\end{array}$ & $\begin{array}{l}100 \\
100\end{array}$ \\
\hline $\begin{array}{l}\text { Opinião do profissional } \\
\text { - O algoritmo é adequado para os fisioterapeutas? }\end{array}$ & 00 & 00 & 13 & 100 & 13 & 100 \\
\hline
\end{tabular}

O aplicativo móvel para diagnóstico multidimensional da vulnerabilidade clínico funcional e tratamento fisioterapêutico em idosos foi criado. O software possui 34 telas que descrevem os procedimentos, a avaliação, o diagnóstico e o tratamento, servindo como ferramenta de apoio nos campos de aplicação em saúde, administração sanitária, doença, assistência médica e terapia diagnóstica. Registrado no Instituto Nacional de Propriedade Industrial (INPI), sob o número BR512020002269-3, marca GERONTOFISIO.

\section{DISCUSSÃO}

Este estudo apresenta a criação de um algoritmo que foi convertido em software para auxiliar fisioterapeutas no processo de tomada de decisões quanto ao diagnóstico multidimensional da vulnerabilidade clínico funcional e tratamento fisioterapêutico em idosos.

No processo de validação do algoritmo, foi demonstrado concordância entre os juízes, e ele foi, portanto, considerado adequado. Este resultado vai de encontro a outros estudos 
encontrados na literatura ${ }^{45,46}$. Embora tenha sido realizado um estudo-piloto, sua confiabilidade e consistência interna serão testadas com a aplicação em uma amostra maior da população no futuro. Através da percepção e vivência dos profissionais fisioterapeutas, foi possível verificar a necessidade de tornar ágil o processo de avaliação.

Isso possibilitou, então, a criação de uma ferramenta tecnológica do tipo aplicativo móvel, identificada como Gerontofisio, de fácil aplicabilidade. Esta ferramenta poderá auxiliar o trabalho fisioterapêutico, essencial para a prevenção de agravos, a promoção da saúde e a reabilitação $0^{47}$. O uso de aplicativos móveis por profissionais de saúde atinge proporções de $45 \%$ a $85 \%{ }^{48}$. Por isso, optou-se no presente estudo pelo método do design centrado no usuário, situado na criação e envolvimento do usuário na concepção de sistemas informatizados ${ }^{49}$.

Diferente do apresentado no presente estudo, mas no mesmo sentido de agilizar os processos de avaliação, Nuñez Filha et al. ${ }^{50}$ desenvolveram um aplicativo móvel para auxiliar os profissionais de saúde na avaliação do risco de quedas do idoso a partir de instrumentos adaptados para o português do Brasil, validados para a população idosa e com amplo uso na literatura. O software foi desenvolvido para dispositivos móveis na plataforma Android, com utilização off-line, assim como o do presente estudo.

Em um estudo de Santos et al..$^{51}$, por exemplo, para melhorar a postura, o equilíbrio e a marcha de idosos, foi criado um aplicativo de exercícios funcionais, com objetivo de orientar e estimular a prática de atividade física, com enfoque em membros inferiores. Já em outro estudo de Santos et al. ${ }^{16}$, foi desenvolvido um aplicativo capaz de identificar com precisão e segurança sinais da síndrome de fragilidade em idosos.

Outros estudos apontam para a criação de aplicativos móveis direcionados aos próprios idosos e apresentam uma relação de apps a essa população com objetivo de orientá-los quanto à prática de atividades físicas, à prevenção de quedas, às atividades da vida diária e aos cuidados ${ }^{15,16}$. Diferente destes aplicativos, o Gerontofisio possui informações que podem ser capazes de detectar vulnerabilidade no aspecto multidimensional e orientar os fisioterapeutas com relação aos objetivos terapêuticos de tratamento com idosos.

A proposta de avaliação da vulnerabilidade no aspecto multidimensional no aplicativo considerou os sinais clínicos para fragilização e declínio funcional.É relevante destacar que a seleção dos itens de avaliação incluiu fatores intrínsecos e extrínsecos adequados, capazes de informar se o idoso está ou não em risco para declínio funcional, vulnerabilidade e/ou fragilidade.
A fisioterapia é peça-chave no envelhecimento ativo e no combate à incapacidade funcional, que influenciam diretamente na qualidade de vida dos idosos. O fisioterapeuta utiliza de seus conhecimentos e recursos para garantir o mais alto padrão de funcionalidade motora e independência física em idosos. Diferentes estudos corroboram com essas afirmações ${ }^{18,52-54}$.

$\mathrm{O}$ aplicativo Gerontofisio pode contribuir para o desenvolvimento de importantes estudos científicos futuros que busquem evidenciar sua eficácia e usabilidade. Portanto, diante dessas informações, este estudo pode ser visto como uma inovação para o envelhecimento e para as áreas de interesse da fisioterapia e da saúde.

Como benefícios deste estudo, os juízes fisioterapeutas adquiriram conteúdos confiáveis para auxiliá-los na tomada de decisões assertivas de maneira rápida frente ao diagnóstico e ao tratamento dos idosos em processo de vulnerabilidade clínico funcional. Em se tratando de fisioterapeutas que trabalham no ramo da gerontologia na sociedade, por meio do uso do aplicativo eles podem aprimorar suas habilidades de abordagem com os pacientes idosos, estabelecer parâmetros para o acompanhamento do paciente e padronizar seus próprios protocolos de avaliação, pois os instrumentos deste estudo foram desenvolvidos com embasamento em evidências identificadas na revisão integrativa da literatura.

A principal limitação do aplicativo móvel Gerontofisio refere-se ao tratamento fisioterapêutico, em que é apresentada a sugestão de condutas de reabilitação no que diz respeito aos objetivos terapêuticos. Embora essa limitação não tenha prejudicado a pesquisa, espera-se desenvolver alternativas para corrigi-la, como a inclusão de recursos audiovisuais com exercícios de cinesioterapia, por exemplo.

Por isso, o aplicativo móvel Gerontofisio pode vir a ser uma opção positiva na área, por fornecer de forma instantânea, clara e gratuita uma avaliação ampla das condições clínicas apresentadas pelo idoso e uma avaliação fisioterapêutica com sugestão de objetivos de reabilitação.

\section{CONCLUSÃO}

O algoritmo foi construído e validado, o software do tipo aplicativo móvel para o diagnóstico multidimensional da vulnerabilidade clínico funcional e tratamento fisioterapêutico em idosos foi desenvolvido, é válido na área de fisioterapia e deve contribuir para fisioterapeutas e pessoas idosas. 


\section{REFERÊNCIAS}

1. Campos MO, Rodrigues-Neto JF, Silveira MF, Neves DMR, Vilhena JM, Oliveira JF, et al. Impacto dos Fatores de Risco para Doenças Crônicas não Transmissíveis na qualidade de vida. Cienc Saude Colet. 2013;18(3):873-82. doi: 10.1590/S1413-81232013000300033.

2. Curzel J, Forgiarini Junior LA, Rieder MM. Avaliação da independência funcional após alta da unidade de terapia intensiva. Rev Bras Ter Intensiva. 2013;25(2):93-98. doi: 10.5935/0103-507X.20130019.

3. Minosso JSM, Amendola F, Alvarenga MRM, Oliveira MAC. Prevalência de incapacidade funcional e dependência em idosos atendidos em um centro de saúde-escola da universidade de São Paulo. Cogitare Enferm. 2010;15(1):12-8. doi: 10.5380/ce.v15i1.17138.

4. Saraiva LB, Santos SNSA, Oliveira FA, Moura DJM, Barbosa RGB, Almeida ANS. Avaliação Geriátrica Ampla e sua Utilização no Cuidado de Enfermagem a Pessoas Idosas. J Health Sci. 2017;19(4):262-7. doi: 10.17921/2447-8938.2017v19n4p262-267.

5. Moraes EN, Carmo JA, Moraes FL, Azevedo RS, Machado CJ, Montilla DER. Índice de Vulnerabilidade Clínico Funcional-20 (IVCF-20): reconhecimento rápido do idoso frágil. Rev Saude Publica. 2016;50(81):1-10. doi: 10.1590/S1518-8787.2016050006963.

6. Piovesan AC, Soares ES, Camillo AA, Corazza ST, Mezzomo SP. Avaliação do Teste de Tinetti e Mini-Exame do Estado Mental em idosas moradoras da comunidade Roberto Binatto, Santa Maria (RS). Rev Kairos Gerontol. 2015;18(1):341-52. doi: 10.23925/2176-901X.2015v18i1p341-352.

7. Alexandre TS, Meira DM, Rico NC, Mizuta SK. Accuracy of Timed Up and Go Test for screening risk of falls among communitydwelling elderly. Braz J Phys Ther. 2012;16(5):381-8. doi: 10.1590/ S1413-35552012005000041.

8. Rebustini RELF, Balbinotti MAA, Filho WJ, Rebustini F, Suemoto CK, Pasqualucci CAG, et al. Validity of the Katz Index to assess activities of daily living by informants in neuropathological studies. Rev Esc Enferm USP. 2015;49(6):944-50. doi: 10.1590/ S0080-623420150000600010.

9. Araújo GKN, Souto RQ, Alves FAP, Sousa RCR, Ceballos AGC, Santos $\mathrm{RC}$ et al. Capacidade funcional e fatores associados em idosos residentes em comunidade. Acta Paul Enferm. 2019;32(3):312-8. doi: 10.1590/1982-0194201900043.

10. Kaushal N, Langlois F, Desjardins-Crépeau L, Hagger MS, Bherer L. Investigating dose-response effects of multimodal exercise programs on health-related quality of life in older adults. Clin Interv Aging. 2019;14(1):209-17. doi: 10.2147/cia.s187534.

11. Moraes GHSM, Cappellozza A, Meirelles FS. Será o fim do papel? os avanços tecnológicos e seus possíveis impactos no consumo de papel. Internext Rev Eletron Negoc Int. 2011;6(2):48-65. doi: 10.18568/1980-4865.6248-65.

12. Oliveira AC, Lucas TC, Iquiapaza RA. O que a pandemia da Covid-19 tem nos ensinado sobre adoção de medidas de precaução? Texto Contexto Enferm. 2020;29(1):1-15. doi: 10.1590/1980-265XTCE-2020-0106.

13. Wu F, Zhao S, Yu B, Chen YM, Wang W, Song ZG, et al. A new coronavirus associated with human respiratory disease in China. Nature. 2020; 579(7798):265-9. doi: 10.1038/ s41586-020-2008-3.

14. Seino S, Kitamura A, Tomina Y, Tanaka I, Nishi M, Nonaka K, et al. Community-Wide Intervention Trial for Preventing and Reducing
Frailty Among Older Adults Living in Metropolitan Areas: Design and Baseline Survey for a Study Integrating Participatory Action Research With a Cluster Trial. J Epidemiol. 2019;29(2):73-81. doi: 10.2188/jea.JE20170109.

15. Amorim DNP, Sampaio LVP, Carvalho GA, Vilaça KHC. Aplicativos móveis para a saúde e o cuidado de idosos. Rev Eletron Comun Inf Inov Saude. 2018;12(1):58-71. doi: 10.29397/reciis.v12i1.1365.

16. Santos TS, Brito TA, Yokoyama-Filho FS, Guimarães LA, Souto CS, Souza SJN, et al. Desenvolvimento de aplicativo para dispositivos móveis voltado para identificação do fenótipo de fragilidade em idosos. Rev Bras Geriatr Gerontol. 2017;20(1):70-6. doi: 10.1590/1981-22562017020.160025.

17. Pereira IM, Bonfim D, Peres HHC, Góes RF, Gaidzinski RR. Tecnologia móvel para coleta de dados de pesquisas em saúde. Acta Paul Enferm. 2017;30(5):479-88. doi: 10.1590/1982-0194201700069.

18. Pillatt AP, Nielsson J, Schneider RH. Efeitos do exercício físico em idosos fragilizados: uma revisão sistemática. Fisioter Pesqui. 2019;26(2):210-7. doi: 10.1590/1809-2950/18004826022019.

19. Moreira WEM, Cassimiro MS, Sales ACM, Pires RLCC, Carvalho PMM. Comparação do Pico de Fluxo Expiratório e a Cirtometria Torácica entre Idosas, Antes e Após a Realização de Fisioterapia Respiratória. Rev Bras Cienc Saude. 2020;24(Supl 2):141-52. doi: 10.22478/ufpb.2317-6032.2020v24nSupl.2.39669.

20. Haynes SN, Richard DCS, Kubany ES. Content validity in psychological assessment: a functional approach to concepts and methods. Psychol Assess. 1995;7(3):238-47. doi: 10.1037/1040-3590.7.3.238.

21. Melnyk BM, Fineout-Overholt E. Evidence-based practice in nursing \& healthcare: a guide to best practice. Philadelphia: Lippincott Williams \& Willkins; 2005.

22. Alexandre NMC, Coluci MZO. Validade de conteúdo nos processos de construção e adaptação de instrumentos de medidas. Cienc Saude Colet. 2011;16(7):3061-8. doi: 10.1590/ S1413-81232011000800006.

23. Hohmann E, Cote MP, Brand JC. Research Pearls: Expert Consensus Based Evidence Using the Delphi Method. Arthroscopy. 2018;34(12):3278-82. doi: 10.1016/j.arthro.2018.10.004.

24. Faller JW, Pereira DN, Souza S, Nampo FK, Orlando FS, Matumoto S. Instruments for the detection of frailty syndrome in older adults: A systematic review. PLoS ONE. 2019;14(4):1-23. doi: 10.1371/journal. pone.0216166.

25. Folstein MF, Folstein SE, McHugh PR. "Mini-mental state": a practical method for grading the cognitive state of patients for the clinician. J Psychiatr Res. 1975;12(3):189-98. doi: 10.1016/0022-3956(75)90026-6.

26. Katz S, Ford AB, Moskowitz RW, Jackson BA, Jaffe MW. Studies of illness in the aged. The index of ADL: a standardized measure of biological and psychosocial function. JAMA. 1963;185(12):914-9. 10.1001/jama.1963.03060120024016.

27. Feitosa-Filho GS, Peixoto JM, Pinheiro JES, Afiune Neto A, Albuquerque ALT, Cattani AC, et al. Atualização das Diretrizes em Cardiogeriatria da Sociedade Brasileira de Cardiologia-2019. Arq Bras Cardiol. 2019;112(5):649-705. doi: 10.5935/abc.20190086.

28. Mendes TAB, Andreoli PBA, Cavalheiro LV, Talerman C, Laselva C. Adequação do uso do oxigênio por meio da oximetria de pulso: um processo importante de segurança do paciente. Einstein. 2010;8(4):449-55. doi: 10.1590/S1679-45082010A01377. 
29. Cordeiro RC, Dias RC, Dias JMD, Perracini M, Ramos LR. Concordância entre observadores de um protocolo de avaliação fisioterapêutica em idosas institucionalizadas. Fisioter Pesqui. 2002;9(2):69-77. doi: 10.1590/fpusp.v9i2.79663.

30. Giaquini F, Lini EV, Doring M. Prevalência de dificuldade de locomoção em idosos institucionalizados. Acta Fisiatr. 2017;24(1):1-6. doi: 10.5935/0104-7795.20170001.

31. Bushatsky A, Alves LC, Duarte YAO, Lebrão ML. Fatores associados às alterações de equilíbrio em idosos residentes no município de São Paulo em 2006: evidências do Estudo Saúde, Bem-Estar e Envelhecimento (SABE). Rev Bras Epidemiol. 2018;21(2):1-14. doi: 10.1590/1980-549720180016.supl.2.

32. Clares JWB, Freitas MC, Borges CL. Fatores sociais e clínicos que causam limitação da mobilidade de idosos. Acta Paul Enferm. 2014;27(3):237-42. doi: 10.1590/1982-0194201400040.

33. Alexandre TS, Duarte YAO, Santos JLF, Lebrão ML. Prevalência e fatores associados à sarcopenia, dinapenia e sarcodinapenia em idosos residentes no Município de São Paulo - Estudo SABE. Rev Bras Epidemiol. 2018;21(2):1-13. doi: 10.1590/1980-549720180009. supl.2.

34. Soukkio P, Suikkanen S, Kaaria S, Kautiainen H, Sipila S, KukkonenHarjula K, et al. Effects of 12-month home based physiotherapy on duration of living at home and functional capacity among older persons with signs of frailty or with a recent hip fracture protocol of a randomized controlled trial (HIPFRA study). BCM Geriatr. 2018;18(232):1-10. doi: 10.1186\%2Fs12877-018-0916-y.

35. Carral JMC, Rodríguez AL, Cardalda IM, Bezerra JPAG. Muscle strength training program in nonagenarians - a randomized controlled trial. Rev Assoc Med Bras. 2019;65(6):851-6. doi: 10.1590/1806-9282.65.6.851.

36. Yamamoto S, Iwata A, Yano Y, Ohmine T, Honma K, Senzaki K, et al. Preliminary study on the effects of movement velocity training of the upper limbs on gait ability in older adults: a nonrandomized controlled trial. Clin Interv Aging. 2019;14(1):781-8. doi: 10.2147/ CIA.S202897.

37. Rodriguez-Larrad A, Arrieta H, Rezola C, Kortajarena M, Yanguas $\mathrm{JJ}$, Iturburu M, et al. Effectiveness of a multicomponent exercise program in the attenuation of frailty in long-term nursing home residents: study protocol for a randomized clinical controlled trial. BMC Geriatr. 2017;17(60):1-10. doi: 10.1186/s12877-017-0453-0.

38. Criekinge TV, Saeys W, Hallemans A, Vereeck L, Hertogh W, Walle PV, et al. Effectiveness of additional trunk exercises on gait performance: study protocol for a randomized controlled trial. Trials. 2017;18(249):1-12. doi: 10.1186/s13063-017-1989-1.

39. Allison SJ, Brooke-Wavell K, Folland J. High and odd impact exercise training improved physical function and fall risk factors in community-dwelling older men. J Musculoskelet Neuronal Interact [Internet]. 2018 [cited 2021 Aug 18];18(1):100-7. Available from: http://www.ismni.org/jmni/pdf/71/jmni_18_100.pdf

40. Jia X, Jiang C, Tao J, Li Y, Zhou Y, Chen L. Effects of core strength training combined with Tai Chi Chuan for the musculoskeletal system and cardiopulmonary function in older adults. Medicine. 2018;97;(35):1-7. doi: 10.1097/md.0000000000012024.

41. Vugt VA, Wouden JC, Bosmans JE, Smalbrugge M, Diest W, Essery R, et al. Guided and unguided internet-based vestibular rehabilitation versus usual care for dizzy adults of 50 years and older: a protocol for a three-armed randomised trial. BMJ Open. 2017;7(1):1-11. doi: 10.1136/bmjopen-2016-015479.

42. Marioni G, Fermo S, Lionello M, Fasanaro E, Giacomelli L, Zanon $S$, et al. Vestibular rehabilitation in elderly patients with central vestibular dysfunction: a prospective, randomized pilot study. Age. 2013;35(6):2315-27. doi: 10.1007/s11357-012-9494-7.

43. Fonseca MA, Cader SA, Dantas EHM, Bacelar SC, Silva EB, Leal SMO. Programas de treinamento muscular respiratório: impacto na autonomia funcional de idosos. Rev Assoc Med Bras. 2010;56(6):642-8. doi: 10.1590/S0104-42302010000600010.

44. Marco E, Coll-Artés R, Marín M, Coll-Fernández R, Pascual MT, et al. Recomendaciones sobre programas de rehabilitación pulmonar en pacientes con enfermedad pulmonar obstructiva crónica de la Sociedad de Rehabilitación Cardiorrespiratoria. Rehabilitación. 2016;50(4):233-62. doi: 10.1016/j.rh.2016.04.004.

45. Cunha DR, Salomé GM, Massahud JMR, Mendes B, Ferreira LM. Construção e validação de um algoritmo para aplicação de laser no tratamento de ferida. Rev Latino-Am Enfermagem. 2017;25(1):1-9. doi: 10.1590/1518-8345.1998.2955.

46. Valim MD, Marziale MHP, Hayashida M, Rocha FLR, Santos JLF. Validade e confiabilidade do questionário de adesão às precauções-padrão. Rev Saude Publica. 2015;49(87):1-8. doi: 10.1590/S0034-8910.2015049005975.

47. Aveiro MC, Aciole GG, Driusso P, Oishi J. Perspectivas da participação do fisioterapeuta no Programa Saúde da Família na atenção à saúde do idoso. Cienc Saude Colet. 2011;16(1):146778. doi: 10.1590/S1413-81232011000700082.

48. Oliveira TR, Costa FMR. Desenvolvimento de aplicativo móvel de referência sobre vacinação no Brasil. J Health Inform [Internet]. 2012 [cited 2021 Aug 18];4(1):23-7. Available from: http://www. jhi-sbis.saude.ws/ojs-jhi/index.php/jhi-sbis/article/view/161/109

49. Barra DCC, Paim SMS, Dal Sasso GTM, Colla GW. Métodos para desenvolvimento de aplicativos móveis em saúde: Revisão Sistemática da Literatura. Texto Contexto Enferm. 2017;26(4):1-12. doi: 10.1590/0104-07072017002260017.

50. Nuñez Filha MCD, Pinto EBC, Leite HJD. Desenvolvimento de um aplicativo para identificação do risco de quedas em idosos. Rev Pesq Fisio. 2018;8(3):354-60. doi: 10.17267/2238-2704rpf.v8i3.2047

51. Santos CMVT, Andrade JA, Amorim AC, Garcia PA, Carvalho GA, Vilaça KHC. Aplicativo em plataforma móvel "Idoso Ativo": exercícios para membros inferiores aliando tecnologia e saúde. Fisioter Mov. 2018;31(1):1-10. doi: 10.1590/1980-5918.031.AO17

52. Ely JC, Fermino AF, Bassani GR, Oliveira HS, Walter J, Amaral SHE, et al. Atuação fisioterápica na capacidade funcional do idoso institucionalizado. Rev Bras Cienc Envelhecimento Hum. 2009;6(2):293-297. doi: 10.5335/rbceh.2012.247

53. Aciole GG, Batista LH. Promoção da saúde e prevenção de incapacidades funcionais dos idosos na estratégia de saúde da família: a contribuição da fisioterapia. Saude Debate [Internet]. 2013 [cited 2021 Aug 18];37(96):10-9. Available from: https:// www.scielo.br/j/sdeb/a/Kr5rYshxg6YPRTLMhcbxvPk/?lang= pt\&format=pdf

54. Gontijo RW, Leão MRC. Eficácia de um programa de fisioterapia preventiva para idosos. Rev Med Minas Gerais. 2013;23(2):173-80. doi: 10.5935/2238-3182.20130028. 
A versão do artigo "Aplicativo móvel: intervenções fisioterapêuticas à idosos frágeis" publicado no volume 28 , número 2,2021 , disponibilizada inicialmente continha erros em relação ao orcid do autor, endereço de correspondência e um erro de tradução na página 222.

\section{Onde se lia:}

\section{ORCID-}

\section{Leia-se:}

ORCID-0000-0002-8287-7476

\section{Onde se lia:}

Rua Aracy Sampaio, no 26 - Pouso Alegre (MG), Brasil - CEP: 18540-000

Leia-se:

Rua Aracy Sampaio, no 26 - Dores de Campos (MG), Brasil - CEP 36213-000

\section{Onde se lia:}

METHODOLOGY

Leia-se:

METODOLOGIA 